\title{
PROGRESS IN ATOM CHIPS AND THE INTEGRATION OF OPTICAL MICROCAVITIES
}

\author{
E. A. HINDS, ${ }^{*}$ M. TRUPKE, B. DARQUIE, J. GOLDWIN and G. DUTIER \\ Center for Cold Matter, Imperial College London, Prince Consort Road, \\ London, SW7 2AZ, UK \\ *E-mail: ab_ed.hinds@imperial.ac.uk \\ http://www3.imperial.ac.uk/ccm/
}

\begin{abstract}
We review recent progress at the Centre for Cold Matter in developing atom chips. An important advantage of miniaturizing atom traps on a chip is the possibility of obtaining very tight trapping structures with the capability of manipulating atoms on the micron length scale. We recall some of the pros and cons of bringing atoms close to the chip surface, as is required in order to make small static structures, and we discuss the relative merits of metallic, dielectric and superconducting chip surfaces. We point out that the addition of integrated optical devices on the chip can enhance its capability through single atom detection and controlled photon production. Finally, we review the status of integrated microcavities that have recently been demonstrated at our Centre and discuss their prospects for future development.
\end{abstract}

Keywords: Atom chip; Integrated optics; Cavity QED; Quantum information.

\section{Introduction}

Atom chips offer a powerful way to miniaturize experiments in atomic physics. ${ }^{1}$ Microstructures on the surface of the chip produce magnetic and electric fields which can be used to confine and manipulate cold atoms above the substrate surface. Experiments have shown that it is possible to transport and split cold neutral atom clouds or Bose-Einstein condensates in tight traps near the surface of a chip using either microscopic patterns of permanent magnetization in a film or microfabricated wire structures carrying currents or charges. Large field gradients can be generated close to a microstructured surface, creating tight traps with oscillation frequencies up to $\sim 1 \mathrm{MHz}$ at the micron scale where tunneling and coupling between traps become important. This makes atom chips suitable for applications in matter-wave interferometry, ${ }^{2}$ quantum sensing, ${ }^{3}$ atomic clocks, ${ }^{4}$ quantum 
information processing, ${ }^{5}$ and the study of low-dimensional quantum gases. ${ }^{6}$ However many of these potential applications require the ability to detect a small number of atoms, to create versatile microscopic trapping configurations, and to manipulate the atomic quantum state, both internal and motional. Optical methods exist to accomplish these tasks, but are only just now starting to be miniaturized and integrated into atom chips.

One microscopic optical device we are studying is an atom detector based on a pair of optical fibres facing each other. These single mode fibres have been machined at their ends, providing small beam waists at the foci. Light transmitted through one fibre and collected by the other should allow detection of 5 to 10 atoms placed in the gap, ${ }^{7}$ either through their absorption (on-resonance imaging) or by the optical phase shift (dispersive imaging). The fibres can also be used to generate a one-dimensional standing-wave pattern, making an optical lattice in which the atoms can be manipulated by the optical dipole force. However, in order to bring the optical sensitivity down to the single atom level, we require an optical cavity to increase the atom-light interaction strength.

The integration of microcavities with the trapping and guiding capabilities of atom chips opens new possibilities for experiments in atomic physics. In an optical microresonator, the interaction strength of an atom with a photon in the cavity mode increases with decreasing mode volume, as $1 / \sqrt{V}{ }^{8}$ This is evident in the expression for the vacuum Rabi frequency in the cavity,

$$
2 g=\mu \sqrt{\frac{\omega_{\mathrm{C}}}{2 \hbar \epsilon_{0} V}},
$$

where $\mu$ is the dipole moment of the atomic transition and $\omega_{\mathrm{C}}$ is the resonance frequency of the cavity. This interaction is damped by the decay rate of the excited atomic population, which is $2 \gamma$ in free space, and of power in the cavity, which is $2 \kappa=\pi c /(L F)$. Here $L$ is the cavity length and $F$ is the finesse of the resonator. The driving and damping rates are succinctly compared in the single atom cooperativity, $C=g^{2} / 2 \kappa \gamma$. The total spontaneous emission rate of an atom in a cavity can be made to differ greatly from the free-space value, depending on the cooperativity. With the cavity tuned to the atomic transition frequency and with $\kappa<g$, the natural decay rate becomes $2 \gamma(1+2 C)$. The second term represents emission of photons into the cavity mode at a rate $4 C \gamma=2 g^{2} / \kappa$.

The resonators used successfully in other experiments have reached single-atom cooperativity values exceeding $50,{ }^{9}$ but are not ideal for atom chip experiments as they consist of comparatively large components, mak- 
ing it impossible to bring the intense part of their mode close to the trapping structures on the chip surface. For this reason, research is being carried out on a variety of integrable microcavity designs. These include microtoroids, fibre-fibre microcavities and our fibre-chip cavities. Microtoroids offer the highest quality factors among these, and are well-suited for scalable atomchip integration as they can be produced by standard silicon microfabrication techniques. Strong coupling between single atoms and the field of a microtoroid resonator has been demonstrated ${ }^{10}$ with atoms passing through the evanescent field in free-fall. To couple light into and out of these resonators, a tapered fibre is used. The fibre position needs to be adjusted so that the evanescent field at the taper overlaps with the evanescent component of the microtoroid mode. This is the only manual procedure required to make the devices operational. While the strong-coupling condition has been experimentally fulfilled for the first time for such a device, the challenge of reliably positioning and trapping atoms in the evanescent field of these devices with the required accuracy has yet to be surmounted.

By contrast, atoms can be placed directly and accurately into the region of highest field strength of Fabry-Perot-type resonators. For this reason, the efforts of several research groups are currently focussed on this type of resonator. Several experimental groups have already succeeded in positioning atoms accurately within the mode of an optical resonator using optical, electrostatic and magnetic transport techniques. The positioning of a Bose-Einstein condensate in a microcavity on a chip by means of current-carrying wire guides has also been recently demonstrated using a fibre-coupled microcavity. ${ }^{11}$ The resonators used in that work are however not ideally suited for scalable integration, as they are constructed in a sequence of mostly manual steps.

Here we report on recent experiments at our Centre on the detection of atoms with on-chip micocavities, whose design aims to combine the advantages of microfabrication and fibre-coupling with the open access of a Fabry-Perot resonator.

In the next section we describe two typical atom chips that are in use in our laboratory to magnetically trap atoms either with current carrying wires or permanent magnet structures. Section 3 deals with detection of atoms and enhanced emission of photons using on-chip microcavities. We conclude with an outlook in Sec. 4. 


\section{Magnetic atom chips}

To give a concrete example of an atom chip, we show in Fig. 1 an interferometer chip $^{7}$ currently being used in our laboratory. The reflective gold surface is used to form a mirror magneto-optical trap (MOT) as a reservoir of pre-cooled atoms. The coils used for the MOT are seen in the figure. The chip consists of a $3 \mu \mathrm{m}$ layer of gold, thermally evaporated onto a silicon substrate, in which wires have been lithographically defined by ion-beam milling. Currents in the wires allow cold ${ }^{87} \mathrm{Rb}$ atoms to be magnetically trapped and split, in a matter-wave analogue of an optical beam splitter. ${ }^{2,3}$ The inset in Fig. 1 is a microscope image showing the four parallel wires at the heart of the interferometer, which have a center-to-center distance of $300 \mu \mathrm{m}$ between the thick outer pair.

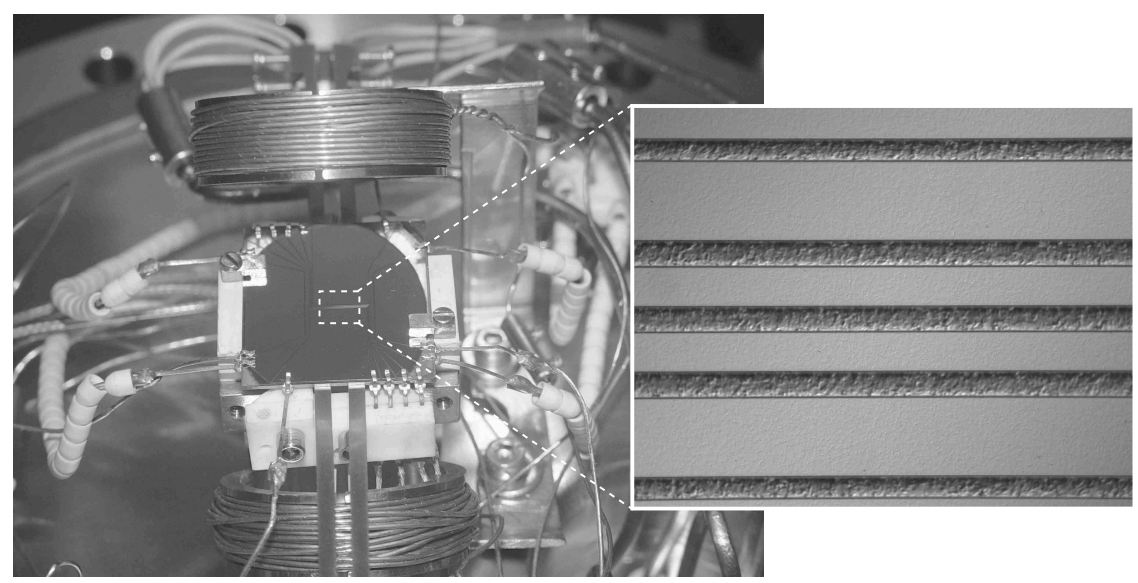

Fig. 1. Current carrying wire atom chip and chip mount used at Imperial College by Eriksson et al. ${ }^{7}$ The inset shows a high resolution microscope image of the central region of the chip where atoms are trapped and manipulated.

With metallic chips such as this one, the homogeneity and stability of cold atom clouds can be compromised close to the room temperature surface. Two main phenomena have been identified: (i) spatial imperfections of the wire cause the current to flow non-uniformly and make the atom trap rough, leading to fragmentation of the atom clouds, ${ }^{12,13}$ and (ii) thermal fluctuations of the magnetic field near the surface, caused by current noise in the conductor, drive spin flips of the atoms, thereby inducing loss and destroying quantum coherences. ${ }^{14}$ The fragmentation can be controlled by using fabrication methods that give very homogeneous wires with smooth 
edges and uniform thickness. The magnetic noise field can be altered by adjusting the thickness of the surface and by changing the material in order to alter the complex conductivity. ${ }^{15,16}$

We have also been using a chip based on patterns of permanent magnetization, written on commercial videotape. This exhibits very much longer spin flip times because the thermal noise currents are suppressed by the high resistivity of the videotape. A pattern of sinusoidal magnetisation (in plane), with a period of about $100 \mu \mathrm{m}$, allows the confinement of atoms at distances less than $100 \mu \mathrm{m}$ from the surface of the chip in an array of long, thin traps. These have a very high aspect ratio $\left(>10^{3}\right)$, with tight transverse confinement that can bring ultra-cold atoms into the one-dimensional regime. ${ }^{17,18}$ As well as offering a long spin-flip lifetime for BECs trapped near its surface ${ }^{17}$ the permanent magnet atom chip has the benefit of low power dissipation. Some fragmentation of these videotape traps has been observed and is due primarily to inhomogeneity of the magnetic layer. Preliminary experiments on $\mathrm{Pt} / \mathrm{Co}$ multilayer magnetic thin films ${ }^{19}$ indicate that these are a promising alternative to videotape. Other permanent magnet materials are being studied in the group of P. Hannaford, also reporting at this confererence.

Superconductors offer another way to reduce the magnetic noise level and increase the spin-flip lifetimes by many orders of magnitude. Indeed, atoms have already been trapped near superconducting surfaces in two laboratories. $^{20,21} \mathrm{~A}$ recent calculation ${ }^{22}$ shows that rubidium atoms trapped $1 \mu \mathrm{m}$ away from a superconducting niobium surface at liquid helium temperature should have a long spin flip lifetime, in excess of $1000 \mathrm{~s}$. Under these conditions, the cold atoms offer a new way to probe the superconducting surface because they are very sensitive to the local magnetic field. For example, cold atoms would be able to image the vortices of a thin superconductor close to the Kosterlitz-Thouless transition or more generally to study vortex dynamics in a type II superconductor. ${ }^{23}$

\section{Chips with optical micro-cavities}

We have recently tested a new type of optical microcavity, which combines the advantages of microfabrication and fibre-coupling with the open access of a Fabry-Perot resonator. The plano-concave resonator is formed between an isotropically etched hemisphere on the surface of a silicon chip and the plane end of single-mode fibre. Both surfaces are coated with a high-reflectivity multilayer dielectric film. Our collaborators at the University of Southampton have fabricated large arrays of concave mirrors on 
silicon chips and we have used them to build cavities ${ }^{24}$ with $F>5000$ and $Q>10^{6}$. More recently, we have demonstrated both the detection of atoms and the enhanced emission of photons into the mode of the cavity. ${ }^{25}$ The apparatus is shown in Fig. 2. Two fibres with plane dielectric mirrors on their ends are held in grooves on a glass-ceramic substrate, facing two of the mirrors in the silicon array. The silicon mirror chip is mounted on a piezo-electric translator (PZT), which is adjusted to tune the upper cavity to the free-space atomic resonance. In this work the lower cavity was not used. A mirror is used to form a reflection MOT directly above the cavities.

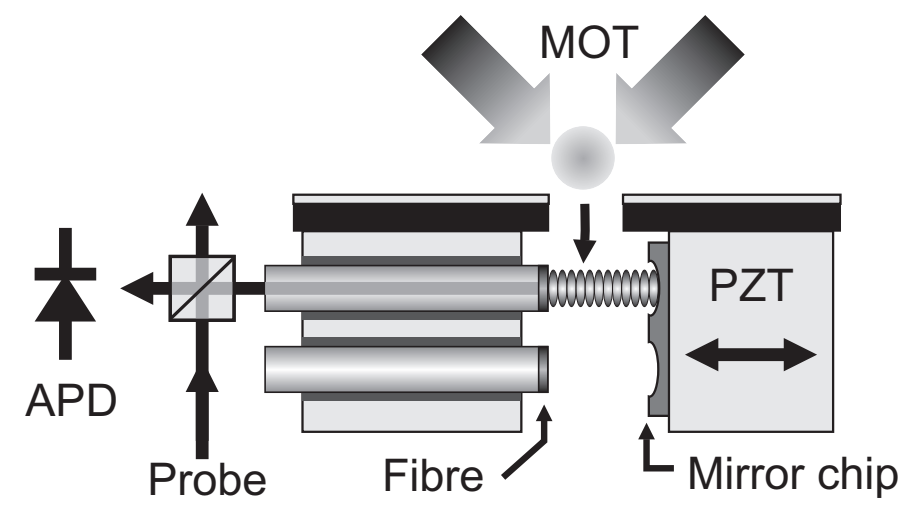

Fig. 2. Schematic diagram of apparatus used in the experiment.

For these experiments, we used cavities with $F=280$ and $L=133 \mu \mathrm{m}$, giving $\kappa=2 \pi \times 2 \mathrm{GHz}$. The beam waist of the mode is $w_{\mathrm{C}}=4.6 \mu \mathrm{m}$, giving a vacuum Rabi frequency of $2 g=2 \pi \times 200 \mathrm{MHz}$ for ${ }^{85} \mathrm{Rb}$ atoms at an antinode of the cavity mode, driven with circularly polarised light on the closed transition $\left|F=3, m_{F}= \pm 3\right\rangle \rightarrow\left|F^{\prime}=4, m_{F^{\prime}}= \pm 4\right\rangle$. This transition has a natural lifetime $2 \gamma=2 \pi \times 6 \mathrm{MHz}$, yielding a single-atom cooperativity of $C=0.8$. The cooperativity increases linearly with the number $N_{\mathrm{A}}$ of atoms in the cavity. Since these are not generally at an antinode, the total cooperativity is $C_{\text {tot }}=\frac{3}{7} C \sum_{n=1}^{N_{\mathrm{A}}} I\left(\mathbf{r}_{n}\right)$ where $I\left(\mathbf{r}_{n}\right)$ is the fraction of peak intensity at the position of the $n^{\text {th }}$ atom. The factor $\frac{3}{7}$ accounts for an average of Clebsch-Gordan coefficients over all the $F=3$ Zeeman sublevels. The effective number of atoms interacting with the cavity field is then defined as $N_{\mathrm{A}}^{\mathrm{eff}} \equiv C_{\text {tot }} /\left(\frac{3}{7} C\right)$. 


\subsection{Atom detection}

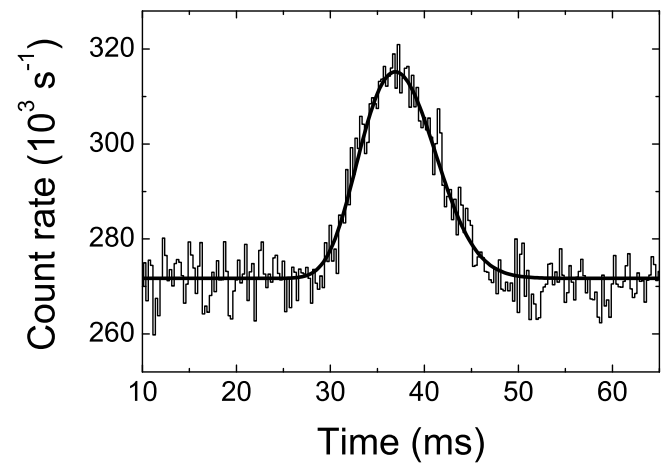

Fig. 3. Change in reflected probe light as atoms traverse the cavity. Laser, cavity, and atom frequencies are equal: $\omega_{\mathrm{L}}=\omega_{\mathrm{C}}=\omega_{\mathrm{A}}$. Average time-of-flight signal of $34 \mathrm{MOT}$ releases, with an integration time of $250 \mu \mathrm{s}$. There are $\sim 0.7$ atoms in the cavity on average at the peak.

To put atoms into the microcavity, we release a cloud of $\sim 2 \times 10^{7}$ ${ }^{85} \mathrm{Rb}$ atoms from the MOT, $7 \mathrm{~mm}$ above. Weak resonant probe light $(\sim$ $1 \mathrm{pW}$ ) is incident on the cavity through a beam splitter and the reflected photons are counted by an avalanche photodiode (APD, see Fig. 2). When the cavity is far from resonance with the light, the reflected intensity is $I_{\max }=419 \times 10^{3} \mathrm{~s}^{-1}$, which drops to $I_{\min }=272 \times 10^{3} \mathrm{~s}^{-1}$ at resonance. As displayed in Fig. 3, this rises to a peak of $I_{\text {atoms }}=315 \times 10^{3} \mathrm{~s}^{-1}$ when the atoms pass through the cavity. From these three count rates we derive the peak cooperativity of the atom-cavity system using the relation

$$
\frac{I_{\text {atoms }}}{I_{\max }} \simeq\left(-1+\frac{v}{P_{\text {tot }}}\right)^{2},
$$

where $P_{\text {tot }}=2 C_{\text {tot }}+1$ is an effective Purcell factor, and $v=1-\sqrt{I_{\min } / I_{\max }}$ is the empty cavity fringe visibility. From our experiments, we determine a total cooperativity of $C_{\text {tot }} \sim 0.23$ at the peak of the atom signal, corresponding to an effective atom number of only $N_{\mathrm{A}}^{\text {eff }}=0.7$.

We have measured the change in the peak reflected intensity when we scan the detuning of the probe light $\Delta_{\mathrm{LA}}=\left(\omega_{\mathrm{L}}-\omega_{\mathrm{A}}\right) / \gamma$, keeping the cavity frequency equal to the atomic resonance frequency $\omega_{\mathrm{C}}=\omega_{\mathrm{A}}$. Figure 4 


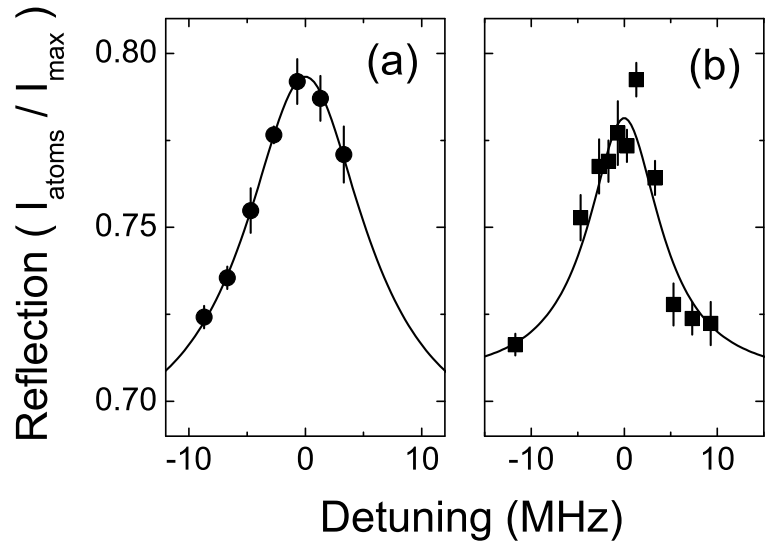

Fig. 4. Peak fraction of power reflected versus detuning $\Delta_{\mathrm{LA}}$ when $\omega_{\mathrm{C}}=\omega_{\mathrm{A}}$ for two different initial MOT numbers. The fits (solid lines) yield average atom numbers of (a) $\left\langle N_{\mathrm{A}}^{\text {eff }}\right\rangle=1.1(\mathrm{~b})\left\langle N_{\mathrm{A}}^{\mathrm{eff}}\right\rangle=0.6 .^{25}$

displays, for two different values of the initial atom number in the MOT, $I_{\text {atoms }} / I_{\max }$ versus $\Delta_{\mathrm{LA}}$, for which we expect

$$
\frac{I_{\mathrm{atoms}}}{I_{\mathrm{max}}} \simeq\left|-1+\frac{v}{P_{\mathrm{tot}}} \frac{1+\Delta_{\mathrm{LA}}^{2}}{1+\Delta_{\mathrm{LA}}^{2} / P_{\mathrm{tot}}+2 i \Delta_{\mathrm{LA}} C_{\mathrm{tot}} / P_{\mathrm{tot}}}\right|^{2},
$$

assuming $g / \gamma \gg\left(1, \Delta_{\mathrm{LA}}\right)$. The solid lines in Fig. 4 are fits to the data, taking into account fluctuations of $C_{\text {tot }}$ and the probe laser linewidth. ${ }^{25}$ These lineshapes confirm that we have better than single atom sensitivity.

\subsection{Noise suppression}

The arrival of atoms in the cavity is signaled not only by an increase in the reflected light level, but also by a decrease in the intensity noise. To measure this, we dropped the cloud 48 times, recording the reflected intensity in $10 \mu \mathrm{s}$ bins. We then determined for each bin the ratio of variance to mean, taken over the 48 drops. After correcting our count rate for the measured APD dead-time of $44 \mathrm{~ns},{ }^{25}$ we found the correct variance-to-mean ratio $f(n) \equiv$ $\operatorname{Var}(n) /\langle n\rangle$ at each time bin $(n$ being the counts already corrected for the dead-time) by including the $10 \%$ loss at the beamsplitter of Fig. 2 and the $60 \%$ loss due to the quantum-efficiency of the APD, according to $f_{\text {corr }}(n)-$ $1=[f(n)-1] / 0.54$. The resulting values, plotted in Fig. 5 , show a strong 


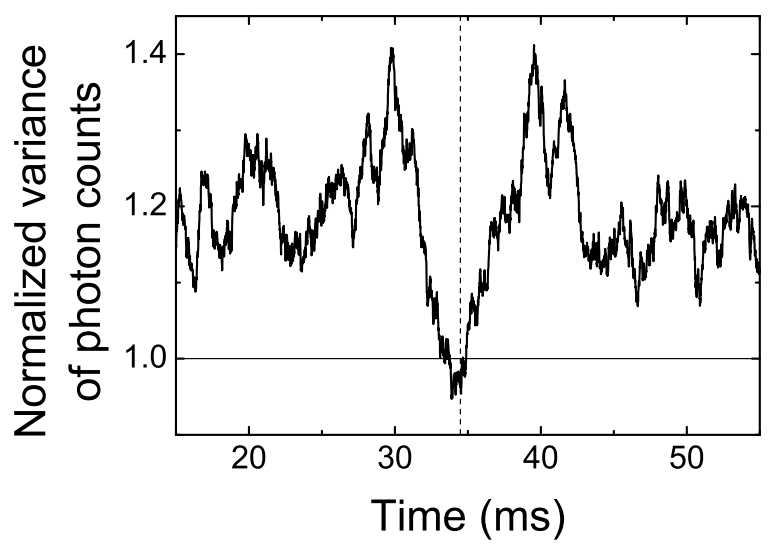

Fig. 5. Ratio of variance to mean for photon count rate reflected from the cavity during passage of atom cloud (corrected for APD dead-time and losses). Vertical dashed line: arrival time of the peak number of atoms. Horizontal line at 1.0: shot noise level. The sample size is 48 atom cloud drops.

reduction of the noise during the passage of the atom cloud. In addition to the photon shot noise, intensity fluctuations are due to small shifts of the cavity length away from its resonance. The effect of these mechanical fluctuations is expected to be less pronounced when atoms are present, and this partially explains the decrease. However many of our experiments seem to show additional noise reduction, the origin of which requires further investigation.

\subsection{Photon generation}

A further effect observed with our microcavity is the enhancement of spontaneous emission. For this experiment, we once again release a cloud of atoms into the resonator. When the atom number in the cavity reaches its highest value, we switch on a resonant excitation beam, aimed at the atoms from below the cavity. This beam pushes the atoms out of the cavity and also pumps them into the dark $F=2$ state. The loss of interacting atoms produces a sharp drop in the cavity reflection signal, as shown in Fig. 6 (a). We then perform the same experiment but without any probe light. This time, a photon peak is recorded at the moment the excitation laser is turned on, as shown in Fig. 6 (b). These are fluorescence photons 


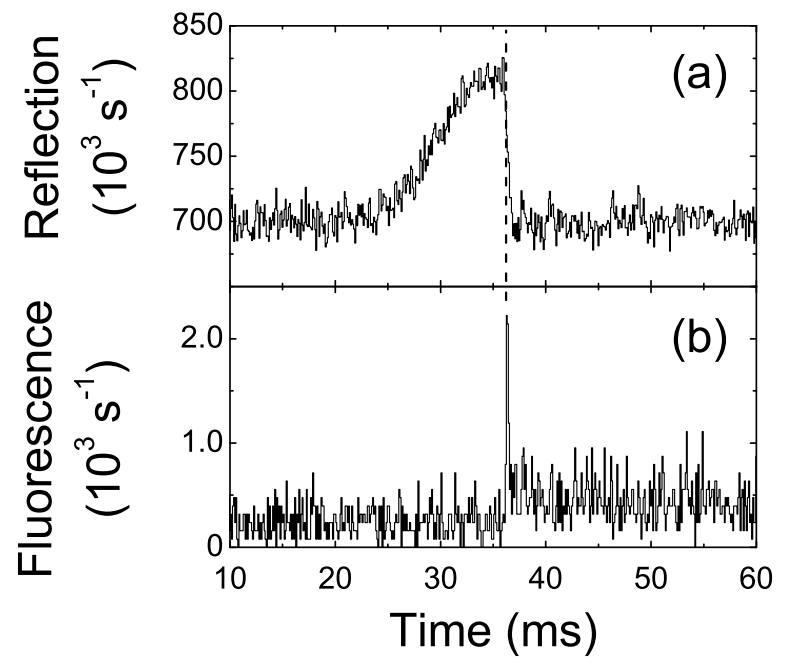

Fig. 6. (a) Sharp drop in the cavity reflection signal due to atom loss and optical pumping when the excitation laser is turned on. (b) Cavity-enhanced spontaneous emission collected via the fiber when the atom cloud is excited. The dotted line shows coincidence of the photon pulse with the turn-on of the excitation laser.

emitted into the cavity mode at the Purcell-enhanced rate $4 C_{\text {tot }} \gamma$, as mentioned in the introduction, and transported to the APD through the fibre. This is confirmed by the fact that no photons are collected when the cavity is detuned from the atomic transition or when there are no atoms in the cavity.

\section{Outlook}

These experiments have shown that this type of microresonator is suitable for the detection of numbers of atoms smaller than one, and can be used to generate photons by enhanced spontaneous emission. The latter is of particular interest for quantum-cryptography and quantum-computation schemes. These cavities have by no means yet reached their full potential. In the laboratory, with materials of the same type, we have created cavities with $F>500$ and $I_{\min } / I_{\max }<15 \%$. Both values are improvements by approximately a factor 2 compared to the published results, which will considerably increase the detection signal, as can be deduced from Eq. (2). We had previously shown that we can even achieve finesse values of $F>5000$, 
albeit with a low fringe-extinction of $I_{\min } / I_{\max } \lesssim 90 \% \cdot{ }^{24}$ At present, these values are limited by scattering losses caused by the surface roughness of the silicon mirror-substrate. Working with the group of M. Kraft at the university of Southampton, we expect in the near future to have smoother cavity mirrors that can operate well within the strong-coupling regime of cavity quantum-electrodynamics, in which the coherent exchange of quantum information between atoms and photons in the cavity becomes possible.

These microcavities have the virtues that they are open, giving atoms direct access to the peak of the mode pattern, they couple the light in and out in a simple way through an integrated fiber, and they can be fabricated in large numbers. Being integrated into a silicon substrate, they can be readily combined with other micromachined components on atom chips. Some examples include the magnetic trapping and guiding structures described in Sec. 2, which allow the deterministic transport of atoms in and out of the cavity, integrated actuators for tuning the resonator, ${ }^{26}$ or pyramidal micro-mirrors to realize an array a of single-atom sources from an array of microscopic MOTs. ${ }^{27}$ Work is in progress to produce a fully integrated device above which neutral atoms can be trapped, guided, detected and manipulated in situ, using magnetic and optical fields.

\section{Acknowledgments}

This work was supported by EU networks Atom Chips, Conquest, and SCALA, by the Royal Society, and by EPSRC grants for QIPIRC, CCM programme and Basic Technology. The atom chip devices in use at Imperial College were fabricated by the nanosystems group of Professor M. Kraft at the University of Southampton.

\section{References}

1. J. Fortagh and C. Zimmermann, Rev. Mod. Phys. 79, 235 (2007).

2. T. Schumm, S. Hofferberth, L. M. Andersson, S. Wildermuth, S. Groth, I. Bar-Joseph, S. J. and P. Krüger, Nat. Phys. 1, 57 (2005).

3. E. A. Hinds, C. J. Vale and M. G. Boshier, Phys. Rev. Lett. 86, 1462 (2001).

4. P. Treutlein, P. Hommelhoff, T. Steinmetz, T. W. Hänsch and J. Reichel, Phys. Rev. Lett. 92, 203005 (2004).

5. T. Calarco, E. A. Hinds, D. Jaksch, J. Schmiedmayer, J. I. Cirac and P. Zoller, Phys. Rev. A 61, 022304 (2000).

6. L. Pricoupenko, H. Perrin and M. Olshanii (eds.), Quantum Gases in Low Dimensions, J. Phys. IV France Vol. 116, (EDP Sciences, 2004).

7. S. Eriksson, M. Trupke, H. F. Powell, D. Sahagun, C. D. J. Sinclair, E. A. 
Curtis, B. E. Sauer, E. A. Hinds, Z. Moktadir, C. O. Gollasch and M. Kraft, Eur. Phys. J D 35, 135 (2005).

8. P. R. Berman (ed.), Cavity Quantum Electrodynamics Advances in Atomic, Molecular and Optical Physics, Supplement 2, (Academic Press, New York, 1994).

9. K. J. Vahala, Nature 424, 839 (2003).

10. T. Aoki, B. Dayan, E. Wilcut, W. P. Bowen, A. S. Parkins, T. J. Kippenberg, K. J. Vahala and H. J. Kimble, Nature 443, 671 (2006).

11. Y. Colombe, T. Steinmetz, G. Dubois, F. Linke, D. Hunger and J. Reichel, arXiv:0706.1390 (2007).

12. M. P. A. Jones, C. J. Vale, D. Sahagun, B. Hall, C. C. Eberlein, B. E. Sauer, K. Furusawa, D. Richardson and E. A. Hinds, J. Phys. B 37, L15 (2004).

13. Z. Moktadir, B. Darquié, M. Kraft and E. A. Hinds, J. Mod. Opt. 54, 2149 (2007).

14. M. P. A. Jones, C. J. Vale, D. Sahagun, B. V. Hall and E. A. Hinds, Phys. Rev. Lett. 91, 080401 (2003).

15. P. K. Rekdal, S. Scheel, P. L. Knight and E. A. Hinds, Phys. Rev. A 70, 013811 (2004).

16. S. Scheel, P. K. Rekdal, P. L. Knight and E. A. Hinds, Phys. Rev. A 72, 042901 (2005).

17. C. D. J. Sinclair, E. A. Curtis, I. L. Garcia, J. A. Retter, B. V. Hall, S. Eriksson, B. E. Sauer and E. A. Hinds, Phys. Rev. A 72, 031603 (2005).

18. C. D. J. Sinclair, J. A. Retter, E. A. Curtis, B. V. Hall, I. L. Garcia, S. Eriksson, B. E. Sauer and E. A. Hinds, Eur. Phys. J. D. 35, 104 (2005).

19. S. Eriksson, F. Ramirez-Martinez, E. A. Curtis, B. E. Sauer, P. W. Nutter, E. W. Hill and E. A. Hinds, Appl. Phys. B 79, 811 (2004).

20. T. Nirrengarten, A. Qarry, C. Roux, A. Emmert, G. Nogues, M. Brune, J.-M. Raimond and S. Haroche, Phys. Rev. Lett. 97, 200405 (2006).

21. T. Mukai, C. Hufnagel, A. Kasper, T. Meno, A. Tsukada, K. Semba and F. Shimizu, Phys. Rev. Lett. 98, 260407 (2007).

22. U. Hohenester, A. Eiguren, S. Scheel and E. A. Hinds, Phys. Rev. A 76, 033618 (2007).

23. S. Scheel, R. Fermani and E. A. Hinds, Phys. Rev. A 75, 064901 (2007).

24. M. Trupke, E. A. Hinds, S. Eriksson, E. A. Curtis, Z. Moktadir, E. Kukharenka and M. Kraft, Appl. Phys. Lett. 87, 211106 (2005).

25. M. Trupke, J. Goldwin, B. Darquié, G. Dutier, S. Eriksson, J. Ashmore and E. A. Hinds, Phys. Rev. Lett. 99, 063601 (2007).

26. C. Gollasch, Z. Moktadir, M. Kraft, M. Trupke, S. Eriksson and E. A. Hinds, J. Micromech. Microeng. 15, S39 (2005).

27. M. Trupke, F. Fernandez-Ramirez, E. A. Curtis, J. P. Ashmore, S. Eriksson, E. A. Hinds, Z. Moktadir, C. Gollasch, M. Kraft, G. V. Prakash and J. J. Baumberg, Appl. Phys. Lett. 88, 071116 (2006). 